OnLine Journal of Biological Sciences 8 (1): 10-14, 2008

ISSN 1608-4217

(C) 2008 Science Publications

\title{
Possibility of High Performance Quantum Computation by using Evanescent Photons in Living Systems
}

\author{
Takaaki Musha \\ MRI, 3-11-7-601, Namiki, Kanazwa-ku, Yokohama 236-0005 Japan
}

\begin{abstract}
Penrose and Hameroff suggested that microtubles in living systems functioned as quantum computers by utilizing evanescent photons. On the basis of the theorem that the evanescent photon is a superluminal particle, the possibility of high performance computation in living systems has been studied. From the theoretical analysis, it is shown that the living system can achieve large quantum bits computation compared with the conventional processors.
\end{abstract}

Key words: Microtuble, Quantum computation, Tunneling photon

\section{INTRODUCTION}

Penrose and Hameroff suggested that microtubles in living systems functioned as quantum computers, with tublin proteins in macrotubles acting as quantum bits of computation ${ }^{[1]}$. Georgiev has supposed that mind is a macroscopic quantum wave governing the dynamics of quantum coherent cytoskeletal protein system inside the cytoplasm of the brain cortical neurons ${ }^{[2]}$. The cytoskeletal protein conformational states are entangled and condensation of evanescent (tunneling) photons emitted by the ordered water that forms coherent domains in its interaction with the local electromagnetic field. Georgiev pointed out that created evanescent photons have negative energy and are shown to be capable of realizing group velocity faster than light velocity in vacuum. It was also proposed by ${ }^{[3-}$ ${ }^{5]}$ that the conscious process in the brain is related with the macroscopic condensates of massive evanescent photons generated by the Higgs mechanism. They claimed that human consciousness can be understand as arising from those creation-annihilation dynamics of a finite number of evanescent photons in the brain. In this article, the author studies the possibility of much higher performance of computation in microtubles of living systems, which utilize evanescent photons compared with solid state computer systems.

The possibility of faster-than-light speed in the quantum region within mictotubles: E. Recami claimed in his study ${ }^{[6]}$ that tunneling photons traveling in an evanescent mode can move with superluminal group speed. The evanescent photon generated in macroscopic domain of the dynamically ordered structure of water satisfies the following Klein-Goldon equation given by:

$$
\left(-\frac{1}{\mathrm{c}^{2}} \frac{\partial^{2}}{\partial \mathrm{t}^{2}}+\nabla^{2}-\frac{\mathrm{m}_{*}^{2} \mathrm{c}^{2}}{\mathrm{~h}^{2}}\right) \mathrm{A}(\mathrm{x}, \mathrm{t})=0
$$

This equation has the solution for the photon traveling in an evanescent mode shown as:

$$
A(x, t)=\exp \left[-\frac{E t+p x}{h}\right]
$$

which corresponds to the superluminal elementary particle of an imaginary mass satisfying $E^{2}=p^{2} c^{2}$ $\mathrm{m}^{2} * \mathrm{c}^{4}$.

By the Faster-Than-Light (FTL) property of evanescent photons, the higher capability of computation by living systems is shown as follows.

Uncertainty principle for the superluminal elementary particle: From relativistic equations of energy and momentum of the moving particle, shown as:

$$
\mathrm{E}=\frac{\mathrm{m}_{0} \mathrm{c}^{2}}{\sqrt{1-\mathrm{v}^{2} / \mathrm{c}^{2}}}
$$

and

$$
\mathrm{p}=\frac{\mathrm{m}_{0} \mathrm{v}}{\sqrt{1-\mathrm{v}^{2} / \mathrm{c}^{2}}}
$$

we obtain the relation given by $\mathrm{p} / \mathrm{v}=\mathrm{E} / \mathrm{c}^{2}$.

From which, we have:

$$
\frac{\mathrm{v} \Delta \mathrm{p}-\mathrm{p} \Delta \mathrm{v}}{\mathrm{v}^{2}}=\frac{\Delta \mathrm{E}}{\mathrm{c}^{2}}
$$


Supposing that $\Delta \mathrm{v} / \mathrm{v}^{2} \approx 0$, Eq. 5 can be simplified as:

$$
\Delta \mathrm{p} \approx \frac{\mathrm{v}}{\mathrm{c}^{2}} \Delta \mathrm{E}
$$

This relation is valid for the superluminal particle, which energy and the momentum can be shown respectively as:

$$
\mathrm{E}=\frac{\mathrm{m}_{*} \mathrm{c}^{2}}{\sqrt{\mathrm{v}^{2} / \mathrm{c}^{2}-1}}
$$

and

$$
\mathrm{p}=\frac{\mathrm{m}_{*} \mathrm{v}}{\sqrt{\mathrm{v}^{2} / \mathrm{c}^{2}-1}}
$$

where $\mathrm{m}_{*}$ is an absolute value of the rest mass for the superluminal particle.

According to M. Park and Y. Park, the uncertainty relation for the superluminal particle can be given by ${ }^{[7]}$.

$$
\Delta \mathrm{p} \cdot \Delta \mathrm{t} \approx \frac{\hbar}{\mathrm{v}-\mathrm{v}^{\prime}}
$$

where $\mathrm{v}$ and $\mathrm{v}^{\prime}$ are the velocities of a superluminal particle before and after the measurement and $\hbar$ is the Plank constant divided by $2 \pi$.

From Eq. 6 and 9 can be rewritten as:

$$
\Delta \mathrm{E} \cdot \Delta \mathrm{t} \approx \frac{\hbar}{\beta(\beta-1)}
$$

when we let $\mathrm{v}^{\prime}=\mathrm{c}$, where $\beta=\mathrm{v} / \mathrm{c}$.

Energy cost for the quantum tunneling photon computation: R. Feynman discussed the possibility of a quantum computer that computational energy cost versus speed is limited by energy dissipation during computation by taking an example of reversible computing ${ }^{[8]}$. According to his idea, the computational speed is limited by minimum energy required to transport a bit of information irreversibly between two devices.

Benioff showed that the computational speed is close to the limit by the time-energy uncertainty principle $^{[9]}$. Margolus and Levitin have also shown that the number of elementary operations that a physical system can perform per second is limited by $2 \mathrm{E} / \pi \hbar$, where $E$ is an averaged energy to perform computation ${ }^{[10]}$. From which the minimum energy to perform computation satisfy the relation $\mathrm{E}_{0} \approx \Delta \mathrm{E}$, where $E_{0}$ is a minimum averaged energy required to perform computation and $\Delta \mathrm{E}$ is an uncertainty of energy to perform computation.

Then we have:

$$
\mathrm{E}_{0} \approx \hbar / \Delta \mathrm{t}
$$

where $\Delta t$ is a operational time of an elementary logical operation.

Instead of the logical gate using particles moving at subluminal speed including photons, energy required for the quantum tunneling photon logical gate becomes:

$$
\mathrm{E}_{0}^{\prime} \approx \frac{\hbar}{\beta(\beta-1) \Delta \mathrm{t}}
$$

As an uncertainty in the momentum of tunneling photons moving at the superluminal speed can be given by:

$$
\Delta \mathrm{p}=\frac{\mathrm{m}_{*} \mathrm{v}}{\sqrt{\mathrm{v}^{2} / \mathrm{c}^{2}-1}}-\frac{\hbar \omega}{\mathrm{c}}
$$

where $\mathrm{m}_{*}$ is an absolute value of the mass for the tunneling photon moving at superluminal speed and $\omega$ is an angular frequency of the photon, the velocity of the tunneling photon can be estimated as ${ }^{[11]}$.

$$
\mathrm{v} \approx \mathrm{c}\left(1+\frac{1}{\sqrt{\omega \Delta \mathrm{t}}}\right)
$$

If we let the tunneling distance be $\mathrm{d}$, the time for a photon tunneling through the barrier can be roughly estimated as by $\Delta \mathrm{t}=\mathrm{d} / \mathrm{v}$. Then the velocity of the tunneling photon can be given by:

$$
\mathrm{v} \approx \mathrm{c}\left(1+\frac{\mathrm{c}}{2 \omega \mathrm{d}}+\sqrt{\frac{\mathrm{c}}{\omega \mathrm{d}}+\frac{\mathrm{c}^{2}}{4 \omega^{2} \mathrm{~d}^{2}}}\right)
$$

If $\mathrm{T}$ is the relaxation time of a single qubit and $\Delta \mathrm{t}$ is the operation time of a single logical gate, the figure of merit of the computation can be defined as $\mathrm{R}=\mathrm{T} / \Delta \mathrm{t}$, which is the number of qubits times the number of gate operations.

As a superposition state of the L-qubits system would decoherence approximately $2^{\mathrm{L}}$ times faster than the superpotition state of one qubit ${ }^{[12]}$, then the relaxation time of the L-qubits system can be roughly estimated to be $2^{-\mathrm{L}}$ times the relaxation time of a single 
qubit computation. Thus the minimum energy required to perform quantum computation for the L-qubits system can be given from Eq. 11 as:

$$
\mathrm{E}_{0} \approx \frac{\hbar v_{\mathrm{G}} \mathrm{L}}{\mathrm{T}} 2^{\mathrm{L}}
$$

where $v_{\mathrm{G}}$ is the number of gate operations.

Similar to this equation, the minimum energy required to perform quantum computation utilizing superluminal particle can be estimated from Eq. 12 as:

$$
\mathrm{E}_{0}^{\prime} \approx \frac{\hbar v_{\mathrm{G}} \mathrm{L}}{\beta(\beta-1) \mathrm{T}} 2^{\mathrm{L}}
$$

Supposing that $\mathrm{E}_{0}=\mathrm{E}_{0}^{\prime}$, an increase of qubit size to perform computation by superluminal evanescent photon compared with the conventional computation can be given by:

$$
\Delta \mathrm{L} \approx \frac{\log _{2}[\beta(\beta-1)]}{1+1 / \mathrm{L} \log 2}
$$

when satisfying $\Delta \mathrm{L}<\mathrm{L}$, where:

$$
\beta \approx 1+\frac{c}{2 \omega d}+\sqrt{\frac{c}{\omega d}+\frac{c^{2}}{4 \omega^{2} d^{2}}}
$$

Possibility of high performance computation in the biological brain: The cytoskelton of biological cells, including neurons of the brain, is made up of microtubles as shown in Fig. $1^{[13]}$. The human brain contains about $10^{18}$ tublins. Each microtuble is a hollow cylindrical tube of tublin proteins, which outer core diameter is $25 \mathrm{~nm}$, as shown in Fig. 2.

Hameroff and Tuszynski proposed that microtuble subunit tublins undergo coherent excitations, which leads to the automatic sequence where quantum coherence superposition is emerged in certain tublins and consciousness is occurred as shown in Fig. $3^{[1]}$. According to their hypothesis of quantum brain, microtuble quantum states link to those of other neurons by quantum coherent photons tunneling through membranes in biological systems functioning in a way resembled as an ion trap computers. In the infra-red spectrum region, evanescent photons they would propagate losslessly in a microtubule as shown in Fig. 4.

From Eq. 18 and 19, an increase of qubit size to perform computation by the evanescent photon compared with the conventional computation, when

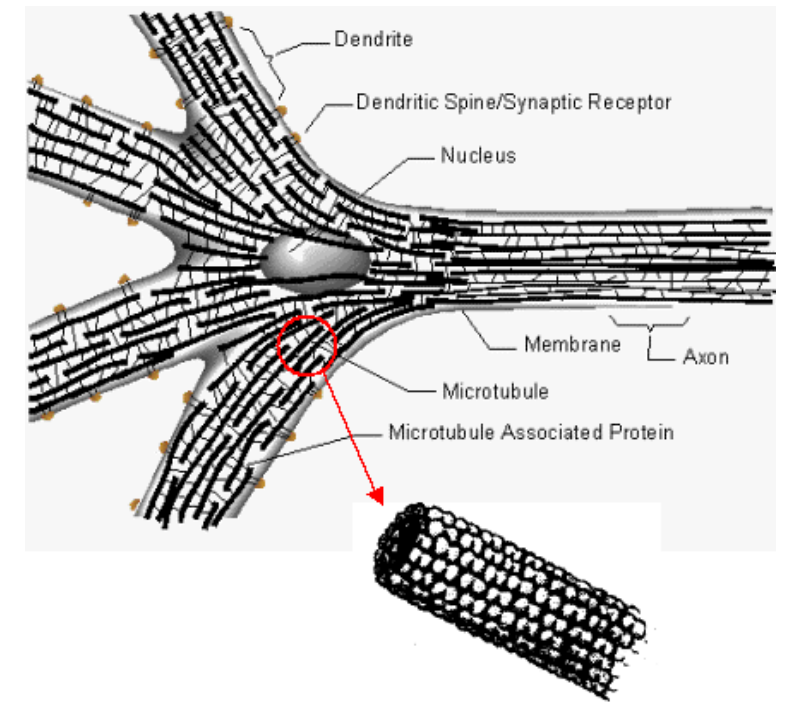

Fig. 1: Cytoskelton of biological cells, including neurons of the brain

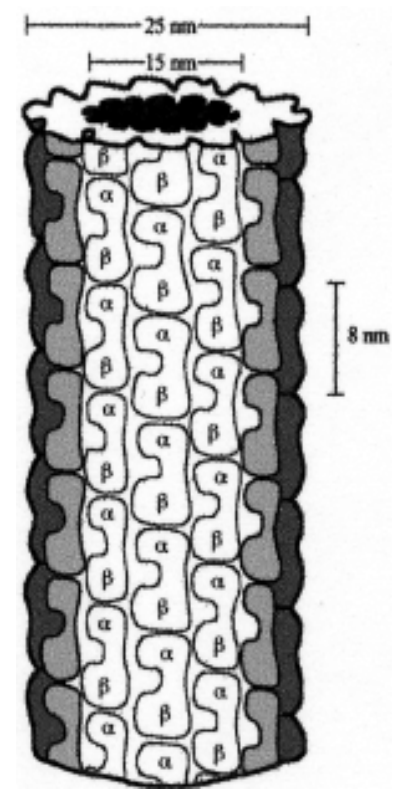

Fig. 2: Hollow cylindrical tube of tublin proteins

satisfying $\Delta \mathrm{L}<\mathrm{L}$, can be obtained as shown in Fig. 5 at the wavelength in the infra-red region, when we let $\mathrm{d}$ $\approx 15 \mathrm{~nm}$, which is of the same order as the extracellular space between the brain cells.

From which, it is seen that the biological brain has the possibility to perform high efficient computation up to 20 qubits more than conventional silicon processors for the infrared light, which wavelength is $\lambda=100 \mu \mathrm{m}$, with the same energy dissipation. 


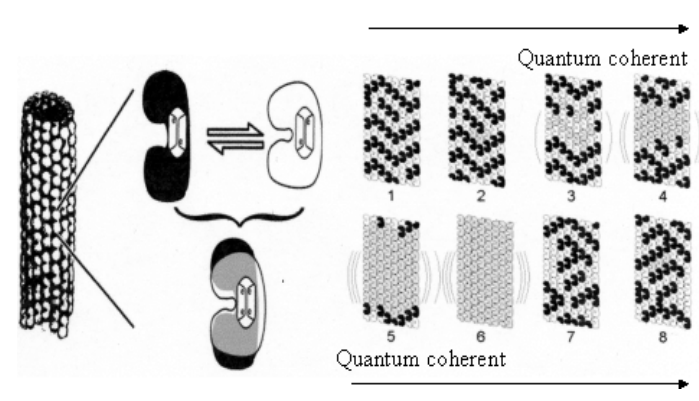

Fig. 3: Schematic diagram of quantum computation conducted in a tuble

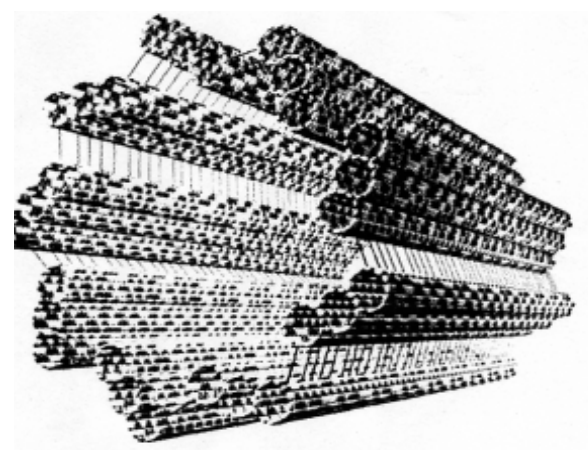

(a) Structure of the centriole cylinder

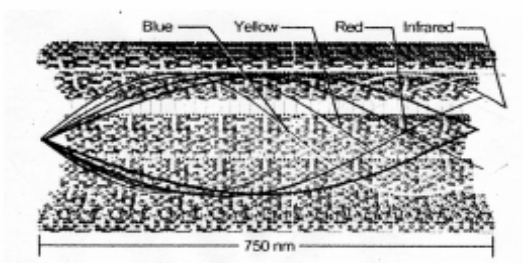

b) Waveguide of visible and infra-red light in the centriole cylinder

Fig. 4: Structure of centriole cylinder comprised of nine microtuble triplets(A) and possible waveguide including visible and infra-red light (B)

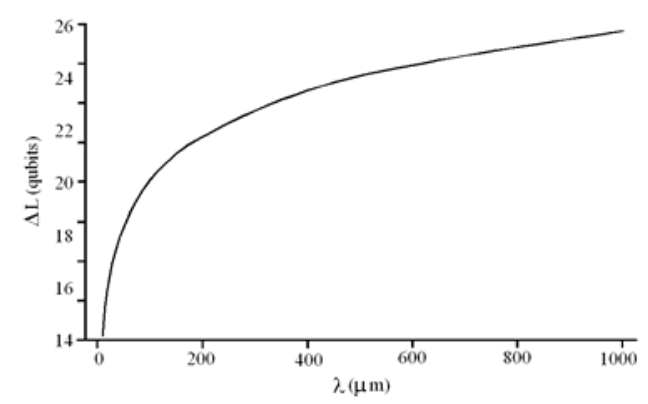

Fig. 5: Increase of quantum bits for quantum computation at the infrared spectrum region
Hence it can be considered that quantum states preserved in microtubules by the superluminal photons attain high efficient computation compared with the silicon processors.

\section{CONCLUSION}

On the basis of the theorem that the evanescent photon is a superluminal particle, the possibility of high performance computation in living systems has been studied. From the theoretical analysis, it is shown that the biological brain has the possibility to achieve large quantum bits computation compared with the conventional processors. Thus it is considered that the human brain has the possibility to attain high efficient computation process compared with silicon processors.

\section{REFERENCES}

1. Hameroff, S. and Penrose, R., 1996. Towards a Science of Consciousness - The First Tucson Discussion and Debate, eds. Hameroff,S.R, Kaszniak, A.W. and Scott,A.C., MIT Press, Cambridge, MA: 507-540.

2. Georgiev, D.D., 2003. On the dynamic timescale of mind-brain interaction, Proceedings of Quantum Mind II: Consciousness, Quantum Physics and the Brain, 15-19 March, The Convention Center, Tucson, Arizona, http://cogprints.org/4463/.

3. Jibu, M. and K. Yasue, 1997. What is mind? Quantum field theory of evanescent photons in brain as quantum theory of consciousness. Informatica, 21: 471-490.

4. Jibu, M., K. Yasue and S. Agan, 1997. Evanescent (tunneling) photon and cellular vision. BioSystem, 42: 65-73.

5. Jibu, M., K.H. Pribram and K. Yasue, 1996. From conscious experience to memory storage and ritrieval: The role of quantum brain dynamics and boson condensation of evanescent photons. Int. J. Modern Phys. B, 10: 1753-1754.

6. Recami, E., 2001. A bird's-eye view of the experimental status-of-the-art for superluminal motions, Found. of Phys. 31: 1119-1135.

7. Park, M. and Y. Park, 1996. On the foundation of the relativistic dynamics with the tachyon. Nuovo Cimento, 111: 1333-1368.

8. Feynman, R.P., 1999. Feynman Lectures on Computation. First edition, Penguin Books, London. 
9. Benioff, P., 1982. Quantum mechanical models of Turing machines that dissipates no energy. Phys. Rev. Lett., 48: 1581-1585.

10. Margolus, N. and L.B. Levitin, 1998. The maximum speed of dynamical evolution. Phys. D, 120: 188-195.

11. Musha, T., 2006. A study on the possibility of high performance computation using quantum tunneling photons. Int. J. Simulation Process Model., 2: 63-66.
12. Gea-Banacloche, J., 2005. Fundamental limit to quantum computation: The energy cost of precise quantum logic, Mathematical Sciences, 508. Saiensu Co.Ltd. Tokyo: 47-57.

13. Hameroff, $\mathrm{S}$ and Penrose.R., 1996. Conscious Events as Orchestrated Space-Time Selections, Journal of Consciousness Studies, 3(1): 36-53. 